\title{
Claudine Friedberg (1933-2018)
}

De l'ethnobotanique à l'anthropologie

Claudine Friedberg (1933-2018): from ethnobotany to anthropology

\section{Serge Bahuchet}

\section{CpenEdition}

\section{Journals}

Édition électronique

URL : http://journals.openedition.org/ethnoecologie/3798

DOI : 10.4000/ethnoecologie.3798

ISSN : 2267-2419

Éditeur

Laboratoire Eco-anthropologie et Ethnobiologie

Référence électronique

Serge Bahuchet, "Claudine Friedberg (1933-2018) », Revue d'ethnoécologie [En ligne], 14 | 2018, mis en ligne le 31 décembre 2018, consulté le 20 avril 2019. URL : http://journals.openedition.org/ ethnoecologie/3798; DOI : 10.4000/ethnoecologie.3798

Ce document a été généré automatiquement le 20 avril 2019

\section{(c)}

Revue d'ethnoécologie est mis à disposition selon les termes de la licence Creative Commons Attribution - Pas d'Utilisation Commerciale - Pas de Modification 4.0 International. 


\title{
Claudine Friedberg (1933-2018)
}

\author{
De l'ethnobotanique à l'anthropologie
}

Claudine Friedberg (1933-2018): from ethnobotany to anthropology

\section{Serge Bahuchet}

$1 \quad$ Née le 16 mai 1933, Claudine Friedberg s'est éteinte le 27 juillet 2018. Avec elle disparaît une personnalité majeure de l'histoire de nos sciences qui explorent les relations entre les sociétés humaines et la nature.

2 Pour rendre compte à la fois des apports décisifs que Claudine Friedberg a fourni à nos disciplines, et de l'itinéraire fascinant d'une personnalité riche au fort tempérament (auxquels nous sommes nombreux à avoir été confrontés, car elle ne mâchait pas ses mots !), je distinguerai quatre étapes significatives. En effet, je ne prétends nullement composer ici une biographie exhaustive, ni dresser une bibliographie complète, mais plutôt présenter une sélection marquante parmi la centaine de ses publications. 
Figure 1 : Claudine Friedberg derrière son bureau rue Cuvier (Juin 2002)

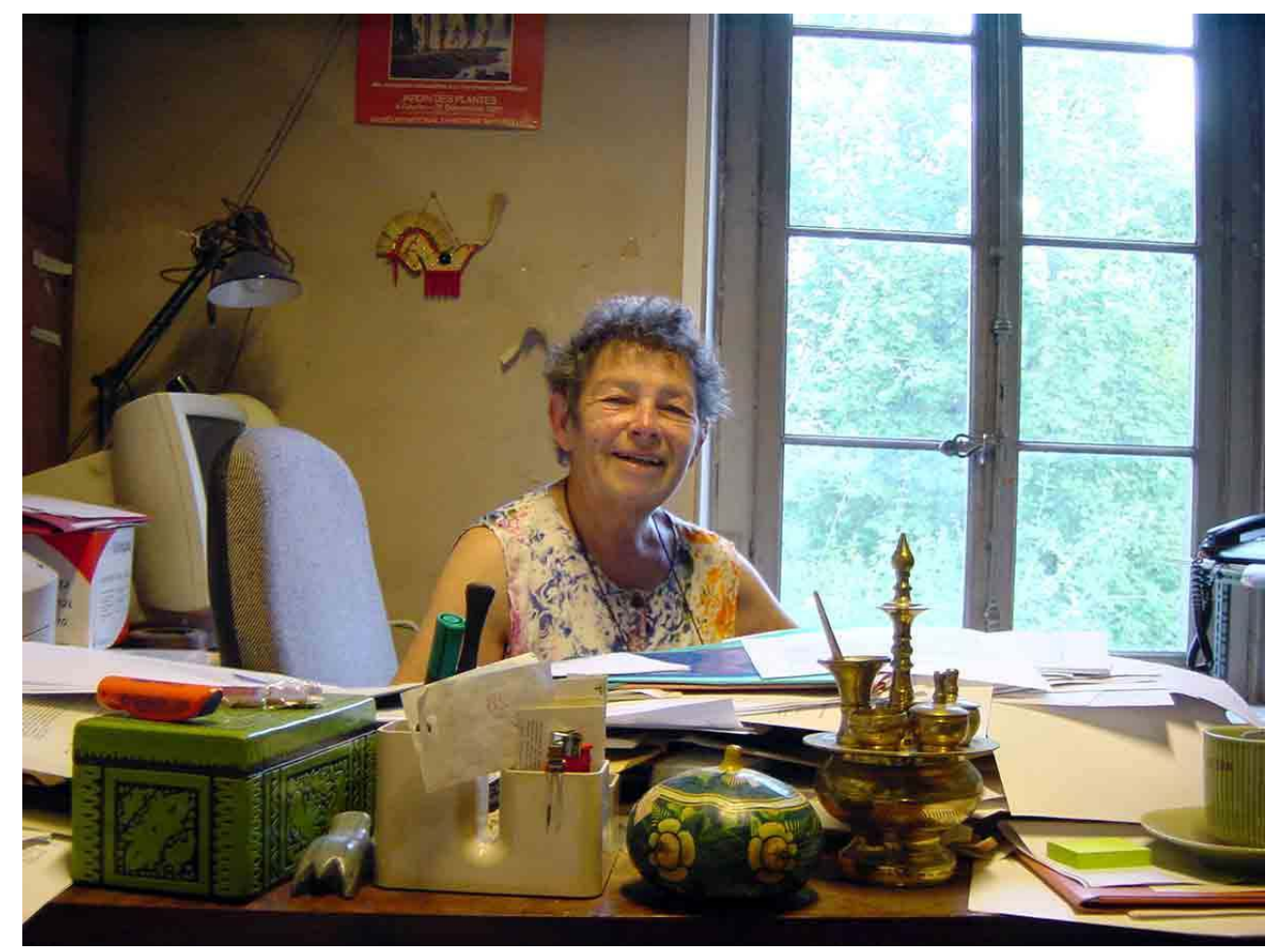

(C) A. Epelboin

\section{Contribution à l'ethnobotanique}

Sur les conseils d'André-Georges Haudricourt, rencontré alors qu'elle suit la formation d'ethnologie du CFRE ${ }^{1}$, Claudine Friedberg se porte candidate en 1956 comme assistante au Muséum ${ }^{2}$, dans le laboratoire d'agronomie tropicale, dont le directeur Roland Portères la recrute pour sa double formation de botaniste et d'ethnologue ( $c f$. le séminaire retranscrit dans Friedberg 2014a). En 1963, Portères transforme sa chaire en «Ethnobotanique » - c'est la première en Europe : nul doute que CF a contribué à cette transformation, en étant la première ethnologue à rejoindre ce nouveau laboratoire composé de botanistes et d'agronomes.

a 23 ans : une frêle jeune femme, dans une institution tri-séculaire majoritairement masculine ; assurément à cette époque, son caractère bien trempé fut une nécessité pour se faire respecter...

Elle applique aussitôt sa double formation à l'étude des « interactions entre les sociétés et les objets naturels, dont elles s'entourent ou dont elles sont entourées », et en premier lieu les plantes (Friedberg 1989a: 2). Elle construit alors des concepts et une méthodologie, fondée sur une rigoureuse détermination des plantes concernées - et donc sur la collecte d'échantillons botaniques. Mais aussi sur une tout aussi rigoureuse transcription de la terminologie vernaculaire (et l'on reconnaît là l'enseignement d'Haudricourt). De ce fait, les collections d'ethnobotanique du MNHN conservent d'elle un herbier géant de plus de 4000 planches, un véritable "monument», car toutes les recherches de CF sont basées sur le concret : la plante. 
Figure 2 : Claudine Friedberg devant son herbier, lors d'un déménagement (Octobre 2009)

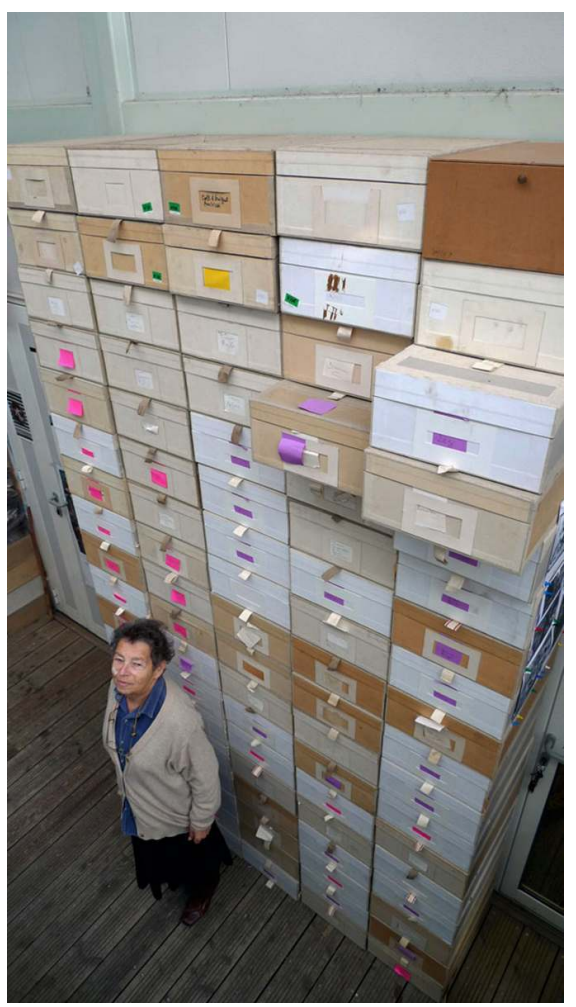

(C) A. Epelboin

6 Le premier terrain de Claudine Friedberg se déroule au Pérou, de novembre 1958 à juillet 1959 (CF 1959b), après qu'elle eut étudié les restes végétaux des fouilles pré-incaïques de H. Reichlen (CF 1958, 1959a). Elle se concentre alors sur les plantes utilisées dans la pharmacopée, qu'elle collecte notamment sur les marchés auprès des herboristes, fascinée qu'elle est de voir l'importance des plantes médicinales dans la vie péruvienne :

« À Lima on peut voir au pied des gratte-ciel, dans une des avenues principales, vendre des racines ou des fleurs séchées réputées pour la guérison de telle ou telle maladie. Dans cette même ville des éventaires plus complets peuvent se rencontrer sur les trottoirs, aux alentours du marché central et au marché 'mayorista' » (CF 1959 : 441). 
Figure 3 : Claudine au Pérou

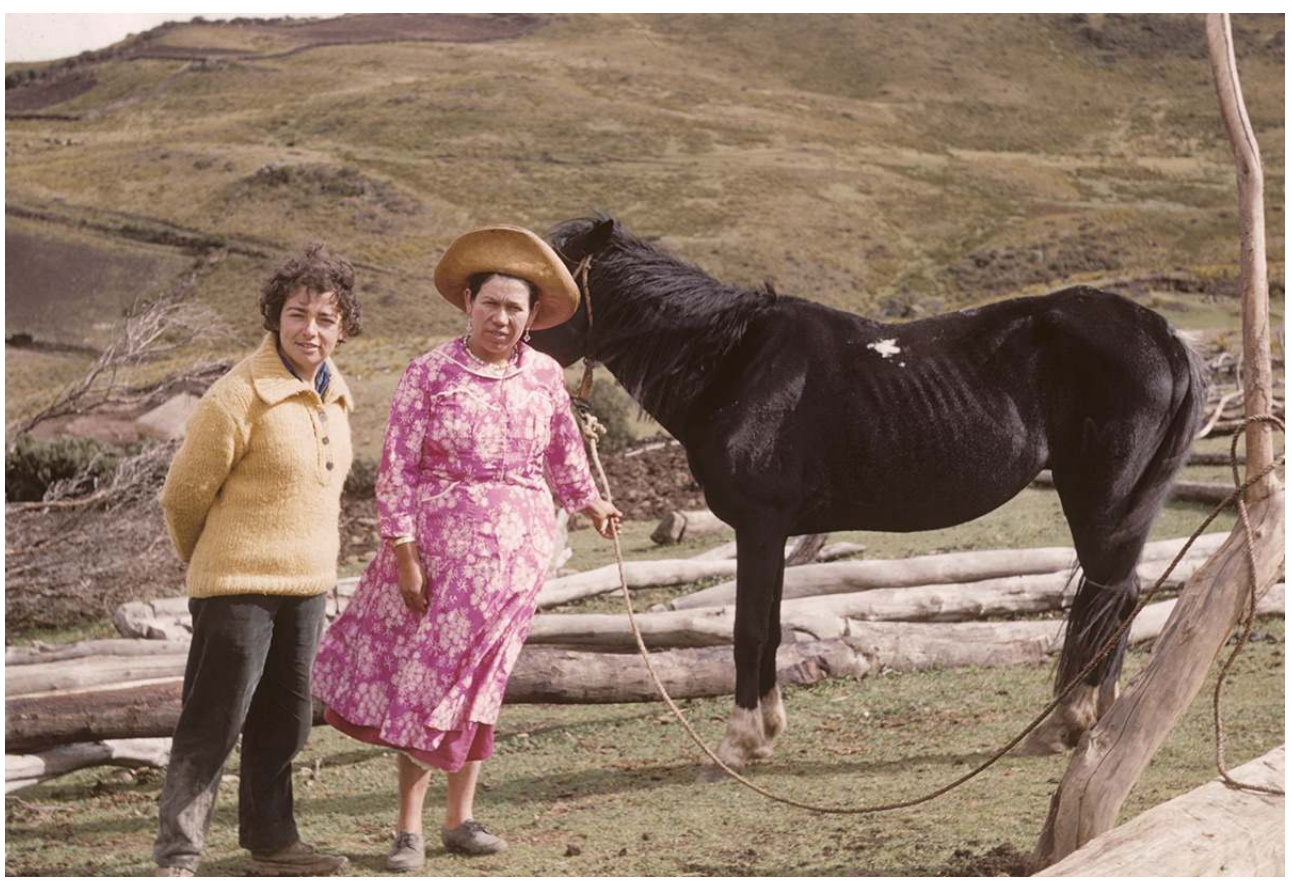

Archives personnelles CF

7 Elle étudie particulièrement l'origine et la circulation de ces plantes et des remèdes d'une région à l'autre du Pérou, et notamment entre les diverses zones climatiques (altitude, forêt, côte...) (CF 1960, 1963a, b, c). Elle sera ainsi amenée à examiner et déterminer les plantes hallucinogènes connues sous le nom d'Ayahuasca, les diverses espèces de Banisteriopsis (Malpighiacées) (CF 1965a, b, c).

Elle reviendra plus tard au Pérou au cours d'une mission de trois mois en 1975 où elle enquêtera notamment sur les guérisseurs maestros (CF 1975), puis en éditant les travaux posthumes de Louis Girault sur les guérisseurs itinérants de Bolivie, à partir des collectes d'ethnobotanique déposées au laboratoire (Girault 1984).

C'est son mariage avec l'ethnologue Louis Berthe qui la conduit en Indonésie, en 1963. L. Berthe (1927-1968) menait alors un travail ethnographique chez les Bunaq de la partie indonésienne de Timor (sur Louis Berthe cf. Pelras 1971), mais les aléas politiques empêchèrent le couple d'atteindre cette région. Le premier séjour se déroula donc à Bali (CF 1963d, 1987b, Friedberg \& Berthe 1963). Ils en rapportent des collections liées aux offrandes, déposées au Musée de l'Homme et maintenant conservées au Musée du quai Branly (cf. Méric 2014). CF y étudiera les offrandes, les plantes médicinales et les traités locaux qui en parlent, sur des feuilles de palmier, et sur les pratiques thérapeutiques liées aux représentations du corps (CF 1985).

En 1966, CF commence son travail à Timor chez les Bunaq pour aborder la classification des plantes dans cette communauté de langue non-austronésienne. Pour ce faire, elle va collecter la totalité des plantes de cet environnement, recueillir tout le savoir tant botanique qu'écologique, et observer toutes les pratiques où des plantes interviennent. Il en résulte une quantité impressionnante de publications, sur le monde des plantes (CF 1970, 1971b, 1972, 1979b, 1990), sur l'agriculture (CF 1971a, 1974a, 1978a), sur l'espace (CF 1973a, b), sur la cuisine (CF 1978c), et sur la société bunaq (CF 1980, 1989b, 2014b). Dans le 
même temps, CF se consacrera à l'édition des textes de littérature orale collectés par L. Berthe (Berthe 1972), qu'elle complètera par ses propres recueils de mythes (CF 1978b) et qui enrichiront sa compréhension du monde bunaq. La synthèse de tous ces travaux fera l'objet de sa volumineuse thèse d'Etat soutenue en 1982 (CF 1982).

Figure 4 : Claudine à Timor

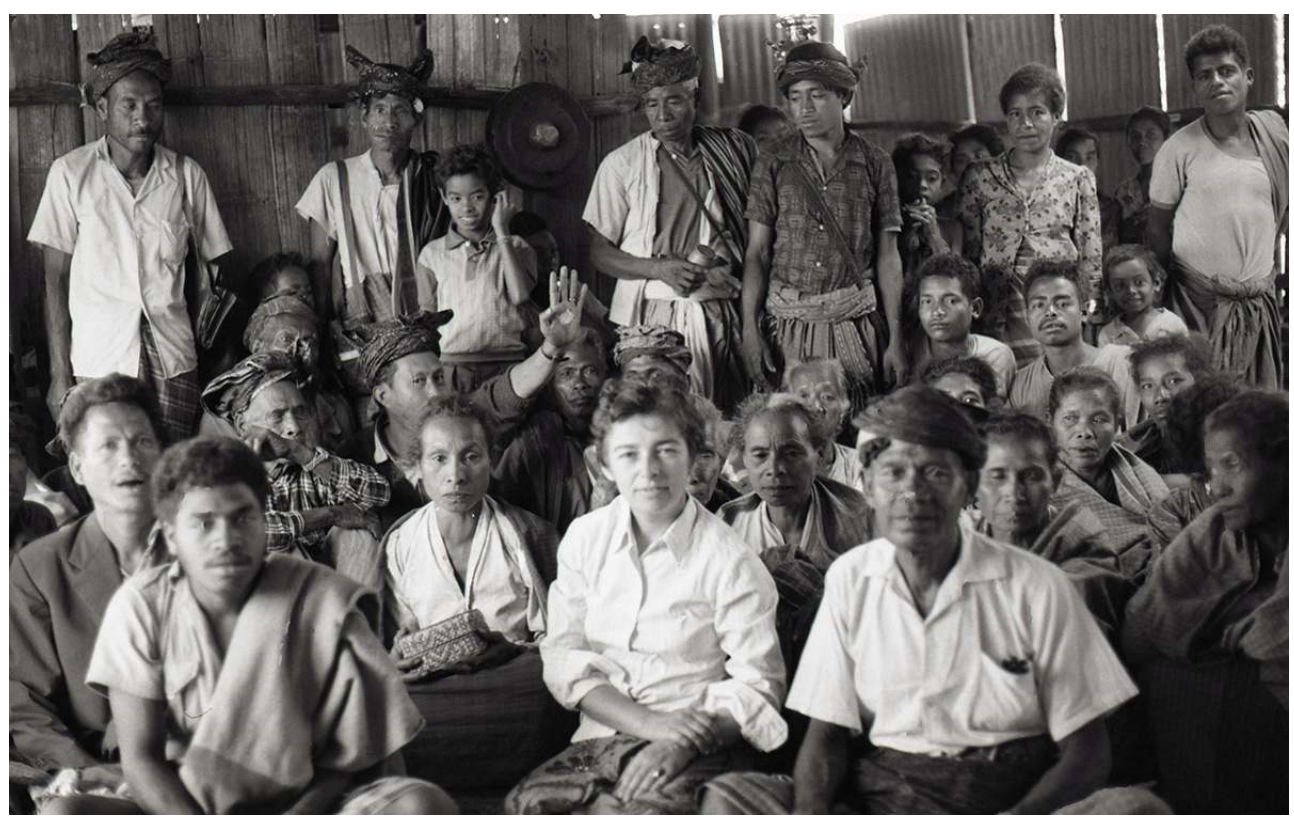

Archives personnelles CF

Par sa démarche originale, fondée par la pratique du terrain, la fréquentation des ethnologues, et une synthèse subtile et toute personnelle entre les enseignements à la fois d'André-Georges Haudricourt, d'André Leroi-Gourhan et de Claude Lévi-Strauss (ce qui est une gageure!), CF modernise l'ethnobotanique classique, en y intégrant la linguistique, notamment pour l'étude des taxonomies vernaculaires, et la nécessité d'y inclure les représentations, les rituels et les structures sociales.

\section{L'étude des processus classificatoires}

12 L'approche de la classification bunaq du monde des plantes est sous-jacente à l'ethnobotanique de CF. Comme elle l'explique elle-même, «ce qui m'intéressait dans l'ordonnancement du monde par les Bunaq était son aspect opératoire dans leur maitrise de l'environnement. » (CF 2014a : 32).

13 Mais sa recherche se déroule alors qu'aux États-Unis d'Amérique se développe une ethnoscience basée sur les Folk classifications, cherchant à dégager des principes universels (Berlin et al. 1973). Ses propres travaux l'amènent à définir des principes et des méthodes, qu'elle affinera en même temps que se précise la nécessité de contester les théories énoncées par les chercheurs états-uniens, et en tout premier lieu Brent Berlin (CF 1968, 1970). La publication de l'œuvre majeure de ce dernier, consacrée aux Tzeltal du Chiapas au Mexique (Berlin et al. 1974), renforcera ses convictions, et entraînera d'autres développements. C'est d'ailleurs la critique du même ouvrage qui provoquera une sorte 
de manifeste de l'ethnobotanique par Marie Martin, du même laboratoire du Muséum (Martin 1975).

14 CF explicite sa démarche comme voulant:

«(...) défendre une vision de l'Ethnoscience héritière d'une conception de la société héritière de l'enseignement de Mauss et de sa notion de 'fait social total ' : on ne peut isoler des domaines culturels et les étudier séparément comme le propose une partie de l'Ethnoscience américaine. Il faut au contraire analyser les articulations entre les différents niveaux de la vie sociale et culturelle. » (CF 1989a :12)

15 À partir donc d'une analyse critique très méticuleuses de deux articles états-uniens sur les classifications, CF explicite sa propre approche et en tire des propositions méthodologiques (CF 1968), notamment l'importance de distinguer :

«1) Système d'identification des plantes et des animaux, 2) système de nomenclature, 3) système de représentation, c'est-à-dire système rendant compte de la façon dont est organisé, au sein d'une culture particulière, l'ensemble du monde naturel. » (CF 1968 : 309)

Elle affine dans l'article suivant (CF 1974b), en contestant la théorie évolutionniste sousjacente dans la théorie de Brent Berlin, et en insistant sur la nécessité de distinguer les mécanismes complexes de ce qu'elle nomme désormais "processus classificatoire », qui « comprend à la fois identification, nomination et insertion dans un système de référence (...) et met en jeu deux mécanismes apparemment contradictoires mais fonctionnant ici de façon complémentaire : différenciation et rapprochement (...)» (1974 : 319). Elle relève également la possibilité de disjonction entre nomenclature, identification et classification, sur la multiplication des systèmes de références au sein d'une même société, et sur le fait important que plusieurs espèces puissent appartenir à plusieurs groupements.

Figure 5 : Couverture de l'ouvrage sur le savoir botanique des Bunaq publié en 1990 par le Muséum national d'Histoire naturelle et tiré de sa thèse

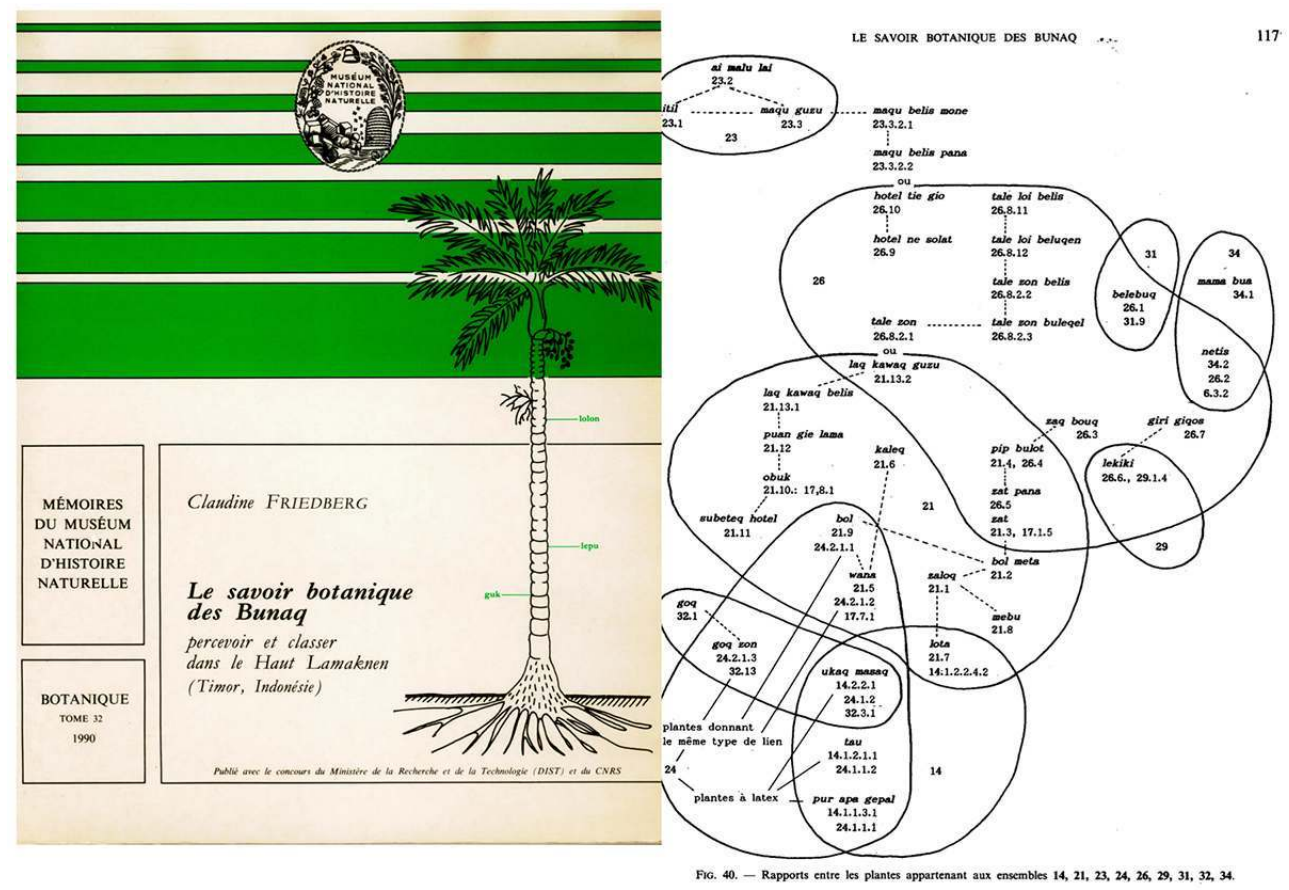

Schéma extrait de l'ouvrage montrant les différents systèmes de référence des classifications chez les Bunaq 
17 Dans plusieurs publications ultérieures, elle posera les principes de l'étude des taxonomies et des processus classificatoires, notamment en introduisant la notion de catégorie complexe, définie par des critères appartenant à des domaines différents (CF 1986, 1991a, b, 1997a, 1999c, d).

\section{Virage anthropologique des ethnosciences}

18 A partir de 1977, et jusqu'en 1983, Claudine Friedberg organise avec Alice Peeters un séminaire très suivi, qui réunit tous ceux qui s'interrogent sur les relations entre sociétés et nature. C'est indéniablement un creuset de réflexion, de débat et de discussion, où se côtoient ethnobiologistes, ethnologues, naturalistes, psychologues, linguistes, chevronnés et étudiants, et où se forgent les idées conduisant à une nouvelle conception de la recherche au sein du laboratoire d'ethnobotanique et d'ethnozoologie ${ }^{3}$.

19 C'est à la même période, de 1977 à 1984, que CF sera membre de la rédaction puis secrétaire générale du JATBA4, (dont notre Revue a pris la suite).

En 1986, CF prend la succession de Jacques Barrau à la tête de l'unité de recherche associée au CNRS «Documentation et recherche en ethnobotanique et ethnozoologie » et la transforme en APSONAT «Appropriation et socialisation de la nature - savoirs, pratiques, représentations » (URA 882 du CNRS). C'est un véritable saut épistémologique, en mettant l'anthropologie au cœur du projet des ethnosciences.

21 CF diffuse ses connaissances dans de nombreux enseignements (à la Sorbonne, à l'EHESS, à Montpellier...) et finalement en contribuant, dès la création en 1995, aux enseignements du DEA interdisciplinaire ETES $^{5}$ (Environnement: temps, espaces, sociétés) associant le MNHN, les Universités Paris I, IV, VII et Orléans, l'IRD et l'INA-PG. Elle y enseigne notamment la catégorisation des objets naturels, et les aspects opératoires des modes de catégorisation en relation avec la gestion de l'environnement ${ }^{6}$.

Elle va alors s'engager, en 1992, dans des recherches collectives et interdisciplinaires dans les Cévennes, sur le Causse Méjean, dans le cadre du programme PIR-EVS (1992-1995) du CNRS, puis du programme "Recréer la nature » du Ministère de l'aménagement du territoire et de l'environnement (1996-2000). Cette recherche qui porte sur les pelouses sèches et leur conservation, aborde des questions cruciales de paysages, d'agriculture et d'élevage, de politique de l'environnement, de conservation de la nature, et enfin du rôle des parcs nationaux (Cohen et al. 1997, Cohen éd. 2003, Friedberg et al. 2000).

À partir de 1987, CF est impliquée dans la conception et la création de la Grande Galerie de l'Evolution ${ }^{8}$, transformation de la vieille galerie de zoologie du Muséum. Elle en est cocommissaire pour l'acte 3 "l'Homme facteur d'Evolution", avec Patrick Blandin (CF 2002b). Son regard aiguisé sur les relations culturelles et civilisationnelles des sociétés humaines avec leur milieu impulsera une orientation précieuse à cette importante partie de la galerie, tant sur le sujet des utilisations des ressources naturelles que sur la domestication, par exemple. C'est au cours de cette entreprise qu'elle forge cette très heureuse expression, programmatique : "l'histoire naturelle de l'homme et l'histoire culturelle de la nature », qui ne sera que très partiellement développée dans la GGE ni totalement, malgré son souhait, dans le nouveau Musée de l'Homme ouvert en $2015^{9}$. 


\section{Les combats pour l'interdisciplinarité} bout de l'intuition initiale de Portères («On peut classer l'ethnobotanique dans les Sciences Humaines.» et plus loin: «L'Ethnobotanique appartient plus au groupe des Sociétés humaines qu'à celui de la Biologie végétale, où elle n'a que faire.» (Portères 1961 : 104).) et la conviction de Haudricourt ( «Il n'y a pas d'ethnosciences, il n'y a que de la bonne ethnologie ", selon son expression orale fréquemment répétée) : il n'y a pas « d'ethno 'machins' » (CF 2005) pour segmenter l'ensemble du vivant et de la société; il n'y a pas d'anthropologie sans la prise en compte du milieu naturel et de tous les êtres vivants. 


\section{BIBLIOGRAPHIE}

\section{Travaux de Claudine Friedberg cités dans le texte}

Friedberg C. 1958 - Contribution à l'étude ethnobotanique des tombes précolombiennes de Lauri (Pérou). JATBA 5 (6-7) : 397-428.

Friedberg C. 1959a - Contribution à l'étude ethnobotanique des tombes précolombiennes de Lauri (Pérou) (Suite et fin). JATBA 6 (8-9) : 405-435.

Friedberg C. $1959 b$ - Rapport sommaire sur une mission au Pérou. JATBA 6 (8-9) : 439-450.

Friedberg C. 1960 - Ethnobotanique péruvienne ; répartition des espèces utilisées par l'homme dans la médecine et la magie et ses rapports avec les zones floristiques. C. R. Soc. Biogéographie, $324: 46-56$

Friedberg C. 1963a - Mission au Pérou (suite) : mai 1961 - mars 1962. JATBA 10 (5-6-7) : 245-258.

Friedberg C. 1963 b - Mission au Pérou : mai 1961 - mars 1962 (suite et fin). JATBA 10 (8-9) : 344-386.

Friedberg C. 1963C - Mission au Pérou : mai 1961 - mars 1962. JATBA 10 (1-4) : 33-52.

Friedberg C. 1963d - Les plantes, les Dieux et les Hommes dans l'île de Bali. Science et Nature 59 : 1-12.

Friedberg C. 1965a - Des Banisteriopsis utilisés comme drogue en Amérique du Sud. Essai d'étude critique. JATBA 12 (9-10) : 403-437.

Friedberg C. 1965b - Des Banisteriopsis utilisés comme drogue en Amérique du Sud. Essai d'étude critique (2e partie). JATBA 12 (11) : 550-594.

Friedberg C. 1965C - Des Banisteriopsis utilisés comme drogue en Amérique du Sud. Essai d'étude critique (Fin). JATBA 12 (12) : 729-780.

Friedberg C. 1968 - Les méthodes d'enquête en Ethnobotanique. Comment mettre en évidence les taxonomies indigènes? JATBA 15 (7-8) : 297-324.

Friedberg C. 1970 - Analyse de quelques groupements de végétaux comme introduction à l'étude de la classification botanique bunaq. In : Échange et Communications, Mélanges offerts à Claude LéviStrauss. Paris, Mouton : 1092-1131.

Friedberg C. 1971a - L'agriculture des Bunaq de Timor et les conditions d'un équilibre avec le milieu. JATBA 18 (12) : 481-532.

Friedberg C. 1971b - Aperçu sur la classification botanique bunaq (Timor central). Bulletin de la Société botanique de France 118 (3-4) : 255-262.

Friedberg C. 1972 - Eléments de botanique bunaq. In : Thomas J.M.C. \& Bernot L. (Ed.), Langues et techniques, nature et société. Paris, Klincksieck : 375-393.

Friedberg C. 1973a - Repérage et découpage du temps chez les Bunaq du centre de Timor. Archipel $6: 119-144$

Friedberg C. $1973 \mathrm{~b}$ - Espace bunaq, pour une exploration naturaliste d'un paysage culturel. In : L'Homme, hier et aujourd'hui, recueil d'études en hommage à A. Leroi-Gourhan. Paris, Cujas : 391-419. 
Friedberg C. 1974a - Agricultures timoraises. Études rurales 53-54-55-56 : 375-405.

Friedberg C. 1974b - Les processus classificatoires appliqués aux objets naturels et leur mise en évidence. Quelques principes méthodologiques. JATBA 21 (10/11/12) : 313-334.

Friedberg C. 1975 - Rapport sur une mission ethnobotanique au Pérou. JATBA 22 (7-9) : 1277-287

Friedberg C. 1978a - The development of traditional agricultural practices in Western Timor. In : Friedman J. \& Rowlands M. J. (Ed.), The Evolution of Social Systems. London, Duckworth and Co. : 137-172.

Friedberg C. 1978b - Comment fut tranchée la liane céleste : et autres textes de littérature orale bunaq (Timor, Indonésie) recueillis et traduits par Louis Berthe. Paris, Société d'Études Linguistiques et Anthropologiques de France (SELAF), 294 p.

Friedberg C. 1978c - La cuisine bunaq. ASEMI, IX (3-4) : 215-227.

Friedberg C. 1979a - Anthropologie, écologie, géographie, ethnoscience. In : L'anthropologie en France : situation actuelle et avenir. Paris, Ed. CNRS : 239-242.

Friedberg C.1979b - Socially significant plant species and their taxonomic position among the Bunaq of Central Timor. In : Ellen R.F. \& Reason D. (Ed.), Classifications in their social context. London, Academic Press : 81-101.

Friedberg C. 1980 - Boiled Woman and Broiled Man: Myths and Agricultural Rituals of the Bunaq of Central Timor. In : Fox J.J. (Ed.), The Flow of Life: Essays on Eastern Indonesia. Cambridge (Mass.) and London, Harvard University Press : 266-289.

Friedberg C. 1982 - Muk Gubul Nor: « la chevelure de la terre » : les Bunaq de Timor et les plantes. Université Paris V-René Descartes, 5 vol., 1857 p.

Friedberg C. 1985 - La santé à Bali : le plein, le tempéré et le juste milieu. L'Ethnographie 96-97 (2-3) : 141-167.

Friedberg C. 1986 - Classifications populaires des plantes et modes de connaissance. In : Tassy P. (Ed.), L'ordre et la diversité du vivant. Paris, Fayard : 22-49.

Friedberg C. 1987a - Les études d'ethnoscience. Le courrier du CNRS 67 : 19-24.

Friedberg C. $1987 \mathrm{~b}$ - Cuisine balinaise : esquisse pour une analyse de la cuisine rituelle de Tenganan Peperingsingan. In : Koechlin B., Sigaut F., Thomas J.M.C. \& Toffin G. (Ed.) De la voûte céleste au terroir, du jardin au foyer : mosaïque sociographique (textes offerts à Lucien Bernot). Paris, EHESS : 383-404.

Friedberg C. 1989a - Titres et travaux de Claudine Berthe-Friedberg. Dactylographié, mars 1989, 57 p. Friedberg C. 1989b - Social relations of territorial management in light of Bunaq farming rituals. Bijdragen tot de taal-, land- en volkenkunde / Journal of the Humanities and Social Sciences of Southeast Asia 145 (4) : 548-563.

Friedberg C. 1990 - Le savoir botanique des Bunaq, percevoir et classer dans le Haut Lamaknen (Timor, Indonésie). Mémoires de Muséum, série botanique, Paris, Muséum National d'Histoire Naturelle, $304 \mathrm{p}$.

Friedberg C.1991a - Operative aspects of folk classification. In : Pawley A. (Ed.), Man and a Half. Essays in Pacific Anthropology and Ethnobiology in Honour of Ralph Bulmer. Auckland, The Polynesian Society, Memoir 48 : 102-109.

Friedberg C. 1991b - Classification ; Ethnoscience ; Hallucinogènes. In : Izard M. \& Bonte P. (Ed.), Dictionnaire de l'ethnologie et de l'anthropologie. Paris, PUF : 153-155, 252-255, 320-321. 
Friedberg C. 1992a - La question du déterminisme dans les rapports homme-nature. In : Jollivet M. (Ed.), Sciences de la nature, Sciences de la société Les passeurs de frontières. Paris, CNRS Editions : $55-68$.

Friedberg C. $1992 \mathrm{~b}$ - Ethnologie, anthropologie : les sociétés dans leurs « natures ». In : Jollivet M. (Ed.), Sciences de la nature, Sciences de la société Les passeurs de frontières. Paris, CNRS Editions : 155-166.

Friedberg C. 1992c - Représentation, classification : comment l'homme pense ses rapports au milieu naturel. In : Jollivet M. (Ed.), Sciences de la nature, Sciences de la société Les passeurs de frontières. Paris, CNRS Editions : 357-373.

Friedberg C. 1996 - Forêts tropicales et populations forestières : quelques repères. NSS 4 (2) : 155-167.

Friedberg C. 1997a - Diversité, ordre et unité du vivant dans les savoirs populaires. NSS 5 (1) : 5-17.

Friedberg C. $1997 b$ - Ethnoscience et sciences cognitives. Journal des anthropologues 70 (1) : 41-49.

Friedberg C. 1999a - Les droits de propriété intellectuelle et la biodiversité : le point de vue d'une anthropologue. NSS 7 (3) : 45-52.

Friedberg C. 1999b - La question de la pérennité des valeurs liées au vivant. NSS 7 (4) : 35-35.

Friedberg C. 1999c - Les savoirs populaires sur la nature. Sciences humaines Hors série 24 : 8-11.

Friedberg C. 1999d - Diversity, order, unity. Different levels in folk knowledge about the living. Social Anthropology 7 (1) : 1-16.

Friedberg C. 2002a - Pour un savoir intégré : quelques réflexions à propos du débat public sur les OGM en France. NSS 10 (1) : 54-58.

Friedberg C. 2002b - La Grande Galerie de l'Évolution (suite) - Séquence finale : nouveau départ? NSS 10 (2) : 99-100.

Friedberg C. 2003 - D'où venons-nous ? Que sommes-nous ? Où allons-nous ? NSS 11 (4) : 404-412.

Friedberg C. 2004 - « Unité et identité de l'Homme »: nouveaux regards. NSS 12 (4) : 434-438.

Friedberg C. 2005 - Ethnoscience et autres ethno « machins » aujourd'hui. Journal de la Société des Océanistes 120 (1) : 27-30.

Friedberg C. 2007a - L'évolution au péril des médias : néocréationnisme et dessein intelligent. NSS 15 (1) : 1-2.

Friedberg C. 2007b - Par-delà le visible. NSS 15 (2) : 167-176.

Friedberg C. 2011 - L'anthropologie mise en cause ? NSS 19 (4) : 377-378.

Friedberg C. 2014a - Anthropologica Acta, les grands témoins : Claudine Friedberg. In : Mathieu N.

\& Schmid A.-F. (Ed.) Modélisation et interdisciplinarité : six disciplines en quête d'épistémologie. Versailles, Editions Quae, collection Indisciplines : 21-60.

Friedberg C. 2014b - Protéger les humains et les non-humains. L'exemple des Bunaq de Lamaknen. Revue d'ethnoécologie 6, DOI : 10.4000/ethnoecologie.1875.

\section{Avec d'autres auteurs :}

Blanc-Pamard C., Deffontaines J.-P. \& Friedberg C. 1992 - Techniques et pratiques : à la jonction du naturel et du social. In : Jollivet M. (Ed.), Sciences de la nature, Sciences de la société Les passeurs de frontières. Paris, CNRS Éditions : 347-355 
Cohen M., Friedberg C. \& Mathieu N. 1997 - Pratiques et représentations de la forêt chez les éleveurs du Causse Méjan. In : Corvol A. (Ed.) La Forêt : perceptions et représentations. Paris, l'Harmattan : 289-302.

Cohen M. (Ed.) 2003 - La brousse et le berger. Une approche interdisciplinaire de l'embroussaillement des parcours. Paris, CNRS éditions, $320 \mathrm{p}$.

Friedberg C. \& Berthe L. 1963 - Note ethnobotanique sur l'utilisation rituelle de quelques riz balinais. JATBA 10 (12) : 612-620.

Friedberg C., Cohen M. \& Mathieu N. 2000 - Faut-il qu'un paysage soit ouvert ou fermé ? L'exemple de la pelouse sèche du causse Méjean. NSS 8 (4) : 26-42.

Muxart T., Blandin P. \& Friedberg C. 1992 - Hétérogénéité du temps et de l'espace : niveaux d'organisation et échelles spatio-temporelles. In : Jollivet M. (Ed.), Sciences de la nature, Sciences de la société Les passeurs de frontières. Paris, CNRS Éditions : 403-425

\section{Comme éditrice :}

Berthe L. 1972 - Bei Gua: Itinéraire des ancêtres, mythes des Bunaq de Timor. Paris, CNRS., collection CEDRASEMI, 531 p. [Friedberg éd.]

Girault L. 1984 - Kallawaya : guérisseurs itinérants des Andes. Recherches sur les pratiques médicinales et magiques. Paris, Ed. de l'ORSTOM, Mémoires n 107, 668 p. [Friedberg éd.]

\section{Autres références citées}

Berlin B., Breedlove D.E. \& Raven P.H. 1973 - General Principles of Classification and Nomenclature in Folk Biology. American Anthropologist 75 :214-242.

Berlin B., Breedlove D.E. \& Raven P.H. 1974 - Principles of Tzeltal plant classification. An introduction to the botanical ethnography of a Mayan-speaking people of highland Chiapas. NY, Academic Press, $660 \mathrm{p}$.

Descola P. 2005 - Par-delà nature et culture. Paris, Gallimard, 624 p.

Hoare C. 2012 - Le JATBA et ses ancêtres. Revue d'ethnoécologie 1, DOI : 10.4000/ethnoecologie.673.

Jollivet M. (Ed.) 1992 - Sciences de la nature, Sciences de la société. Les passeurs de frontières. Paris, CNRS Éditions, $589 \mathrm{p}$.

Martin M.A. 1975 - L'ethnobotanique, science per se ? JATBA 22 : 237-276.

Méric A. 2014 - Offrandes et objets rituels collectés par Louis Berthe et Claudine Friedberg à Tenganan Pegeringsingan (Bali) : la collection du musée du quai Branly. Note de recherche, Paris, Musée du quai Branly, $45 \mathrm{p}$.

Pelras C. 1971 - Louis Berthe, 1927-1968. Archipel 1 : 97-101.

Portères R. 1961 - L'ethnobotanique : place - objet - méthode - philosophie. JATBA 8 (4-5) : 102-109.

\section{NOTES}

1. Centre de formation aux recherches ethnologiques, fondé par André Leroi-Gourhan, dispensé à l'Institut d'Ethnologie au Musée de l'Homme. Licenciée en sciences (sciences naturelles et ethnologie-sciences) à l'Université de Paris en 1955, CF sera diplômée du CFRE en 1956. 
2. Assistante au MNHN en 1956, CF sera Maître-assistante en 1972, Sous-directeur en 1989, puis Professeure en 1992.

3. Parmi les participants à ce séminaire, l'un d'entre eux, Philippe Descola, accèdera au Collège de France dans une chaire intitulée « Anthropologie de la nature » (Descola 2005 - voir l'important compte-rendu qu'en fait CF en 2007b).

4. JATBA : Journal d'agriculture traditionnelle et de botanique appliquée: travaux d'ethnobotanique et d'ethnozoologie (Sur l'histoire et les titres successifs du JATBA, voir Hoare 2012).

5. Le DEA ETES deviendra EMTS (Environnement, milieux, techniques, sociétés), puis EDTS (Environnement : dynamique des territoires et sociétés) qui sera une spécialité de master en deux années, dans le cadre du Master du Muséum (en association avec AgroParisTech pour le M2), jusqu'à l'année universitaire 2018-2019.

6. CF a également enseigné à l'Université nationale autonome du Mexique (UNAM) en 1981, et en Indonésie, à partir de 1985, où elle a encadré plusieurs étudiants dans le cadre du LIPI, Institut national de la recherche indonésienne (programme de coopération franco-indonésienne ESIOP Études des Sociétés de l'Indonésie Orientale et Périphérique). Eko Baroto Walujo, Dyah Maria Suharnot, Markus Pattinama et Yohanes Purwanto ont réalisé leur thèse de doctorat sous la direction de CF.

7. PIR-EVS : Programme interdisciplinaire de recherche « environnement, vie, sociétés »

8. La GGE ouvre en 1994.

9. «Voici un beau programme qui permettrait de réunir en un même lieu l'histoire naturelle de l'homme et l'histoire culturelle de la nature et, reprenant l'intention première de cette séquence, de sensibiliser le public à ses responsabilités dans le devenir de la planète. " (CF 2002b : 100, à propos de la rénovation de la GGE et de l'éventuel futur Musée de l'Homme)

10. PIREN : Programme interdisciplinaire de recherche sur l'environnement, du CNRS, créé en 1978.

11. Le livre collectif des Passeurs de frontières rassemble les principaux «militants» de l'interdisciplinarité entre Natures et Sociétés (chercheurs ayant travaillé dans les programmes PIREN, chercheurs de l'INRA-SAD, chercheurs - dont CF - ayant fait partie du séminaire interdisciplinaire de M. Godelier et $\mathrm{O}$. Dolfuss à l'EHESS...). Le séminaire a duré plus de deux ans, à raison d'une séance par mois, réunions où le travail était réparti et par disciplines et par thèmes (mots clés), animées par M. Jollivet pour les sciences sociales, et J.-M. Legay côté sciences de la vie. Évidemment les disciplines les plus interrogées car, comme le disait Claudine, « interdisciplinaires » étaient l'anthropologie et la géographie (comm. pers. de N. Mathieu).

\section{AUTEUR}

\section{SERGE BAHUCHET}

Professeur d'ethnobiologie du Muséum national d'Histoire naturelle, laboratoire Ecoanthropologie et ethnobiologie(MNHN-CNRS-Paris Diderot) 